\title{
ALL FINITE GROUPS ACT ON FAKE COMPLEX PROJECTIVE SPACES
}

\author{
SHMUEL WEINBERGER
}

(Communicated by Haynes R. Miller)

Abstract. We prove the result asserted in the title.

The object of this note is to prove the assertion of its title in a slightly more precise form that shows that one can somewhat control the normal representation at a fixed point and that there is an infinite amount of choice for the rational Pontrjagin classes. This result is in sharp contrast to a conjecture of Ted Petrie [5] that only homotopy $\mathbf{C} P^{n}$,s with the same Pontrjagin classes as standard $\mathrm{C} P^{n}$ can have smooth $S^{1}$ actions. Our method, relying on the surgery theory of Browder and Quinn, implies nothing about his case. This should not be viewed as support for the conjecture since the method also fails in the PL locally linear case, for which counterexamples have been produced in profusion [2]. Many cases of our theorem have been proven, in some cases in a more precise form; in particular M. Hughes has proven the assertion of our title for all odd order groups by a different method. See [3,4] and the references given there.

Definition. The graph of a group action has as labeled vertices the components of fixed sets of the subgroups that label the subgroup, and as edges maps induced by inclusions.

If all components are simply connected and have codimension at least three in one another, the Browder-Quinn surgery group of the situation [1] just depends (modulo dimensions mod 4) on the graph of the action. We call this hypothesis $H$.

Proposition. For any graph there is an $N$, such that if $G$ acts smoothly on $\mathbf{C P}{ }^{n}$, $n \geq N$, with given graph and satisfying hypothesis $H$, then $G$ acts smoothly on infinitely many homotopy $\mathbf{C} P^{n}$ 's in an equivariantly homotopy equivalent way, and with the same normal representations.

Received by the editors September 7, 1987.

1980 Mathematics Subject Classification (1985 Revision). Primary 57S17, 57S30.

Key words and phrases. Transformation groups, surgery.

The author is partially supported by an NSF grant, a Presidential Young Investigator award, and a Sloan Foundation fellowship. 
The reader should note that $H$ can often be eliminated in practice. In particular, by taking the projectivization of any representation $\oplus$ trivial representation we obtain the following corollary.

Corollary. For any representation there are infinitely many homotopy $\mathbf{C} P^{n}$ 's with smooth $G$-action and given representation as the normal representation at some fixed point.

The idea of the proof is to examine the surgery exact sequence of [1] and compare the ranks of the normal invariant set and the surgery obstruction group, and when the former is larger get nontrivial elements in the kernel. To do this we need a group structure on the normal invariant set for which the surgery obstruction map is a homomorphism. We achieve much less, but it is adequate for the present purposes.

Lemma. If $f: M / G \rightarrow \sum X$ is any map, the Browder-Quinn surgery obstruction map is a homomorphism restricted to the subgroup of $[M / G: F / O]$ lying in the image of $\left[\sum X: F / O\right]$.

An analogous statement appears in [6]; it is a trivial consequence of the definition of surgery groups in [6, Chapter 9] which applies whenever a $\pi$ $\pi$ theorem holds. (If $X$ is a submanifold and the map is the collapse map, then one uses the additivity of the surgery obstruction under "stacking." A transversality argument reduces the general case to this one.) There is such a theorem in Browder-Quinn theory [1].

Note that for any finite group action on $\mathbf{C} P^{n}$ the quotient map induces an isomorphism on $[: G / O] \otimes \mathbf{Q}$. It is a classical result.in homotopy theory that the quotient of any space by its middle skeleton is homotopy equivalent to a suspension (essentially by inductively desuspending the attaching map of cells, using Freudenthal), so that, by the lemma, on the subgroup consisting of all normal invariants which are trivial on the middle skeleton, surgery obstruction is a homomorphism. If $n$ is larger than four times the rank of the relevant surgery group there will be elements with vanishing obstruction (by comparing the ranks of these Abelian groups), which then lift to fake $\mathbf{C} P^{n}$ 's with nontrivial rational normal invariant.

Note. This argument is clearly inefficient. Can one at least halve the dimension bound? This is definitely the case for tame topological actions in the sense of [6], a condition much stronger than the now standard locally linear. Some papers of Dovermann (see [3]) might be relevant for the smooth case.

Added in proof. We can now prove the improved dimensional bound.

\section{REFERENCES}

1. W. Browder and F. Quinn, A surgery theory for G-manifolds and stratified sets, Manifolds, University of Tokyo Press, Tokyo, 1973, pp. 27-36.

2. S. Cappell and S. Weinberger, A geometric interpretation of Siebenmann's periodicity phenomenon, Geometry and Topology; Manifolds, Varieties, and Knots, Marcel Dekker, New York, 1987, pp. 47-52. 
3. K. H. Dovermann, Cyclic group actions on complex projective spaces, preprint.

4. M. Hughes, Thesis, Rutgers University, 1987.

5. T. Petrie, Smooth $S^{1}$ actions on homotopy complex projective spaces and related topics, Bull. Amer. Math. Soc. 78 (1972), 105-153.

6. C. T. C. Wall, Surgery on compact manifolds, Academic Press, New York, 1970.

Department of Mathematics, University of Chicago, Chicago, Illinois 60637 